\title{
POREĐENJE UTICAJA KONSTANTNE I PROMENLJIVE BITSKE BRZINE NA BROJ TV KANALA KOD DVB-T2 STANDARDA
}

\author{
Jelena Stojković1, \\ Branimir Jakšić1*, \\ Mile Petrović, \\ Petar Spalević2, \\ Bojan Prlinčević 3
}

\author{
${ }^{1}$ Fakultet tehničkih nauka, \\ Univerzitet u Prištini, \\ Kosovska Mitrovica, Srbija \\ 2Univerzitet Singidunum, \\ Beograd, Srbija \\ ${ }^{3}$ Visoka tehnička škola, \\ Zvečan, Srbija
}

Odgovorno lice:

Branimir Jakšić

e-pošta:

branimir.jaksic@pr.ac.rs

\begin{abstract}
Rezime:
U ovom radu je izvršena analiza uticaja modulacionih šema i kodnog količnika na broj TV kanala u jednom UHF kanalu primenom DVB-T2 standarda. Najpre je korišćenjem DVB kalkulatora određena bitska brzina jednog UHF kanala za različite modulacione formate (QPSK, 16QAM, 64QAM, 256QAM) i kodne količnike. Zatim je određen broj TV kanala (SDTV, HDTV i UHDTV) koji se smešta u jednom multipleksu UHF kanala korišćenjem MPEG-2, MPEG-4 i HEVC kompresionog standarda. Grafički je prikazan broj TV kanala kada se koristi promenljiva i kada se koristi konstantna bitska brzina. Na kraju je dat tabelarni pregled sa parametrima i realnim opterećenjem DVB-T/T2 multipleksa u Srbiji i zemljama u regionu.
\end{abstract}

Ključne reči:

konstantna bitska brzina, promenljiva bitska brzina, DVB-T2, SDTV, HDTV.

\section{UVOD}

Za prenos digitalnog signala u sistemima digitalne zemaljske televizije radio putem, u evropskim zemljama kao i u drugim pojedinim delovima sveta, koristi se DVB-T (Digital Video Broadcasting - Terrestrial) standard prve generacije sistema, koji je do pre skoro čitave decenije bio jedini standard koji se koristio u svim operativnim mrežama digitalne zemaljske televizije širom Evrope. [1]. Kasnije su razvijeni i usvojeni znatno uspešniji standardi za prenos i kodovanje, a osiguran je i prostor za poboljšanje efikasnosti i povećanje kapaciteta DTT mreža [2, 3]. Druga generacija DTT sistema (Digital Terrestrial Television) nazvana DVB-T2 (Digital Video Broadcasting - Second Generation Terrestrial) odlikuje se velikim brojem osobina poboljšanih u odnosu na osobine DVB-T sistema uključujući veći kapacitet, odnosno veću bitsku brzinu unutar radio kanala (približno za 50\% u poređenju sa prvom generacijom DVB-T) [4]. DVB-T2 je uveo nove mehanizme koji poboljšavaju kompaktnost sistema i u zavisnosti od servisnih zahteva omogućio je nivoe zaštite koji se mogu kombinovati [4]. Kod digitalne zemaljske televizije koriste se modulacioni formati: QPSK (Quadrature Phase-Shift Keying) i QAM (Quadrature Amplitude Modulation) u formama 16QAM, 64QAM i 256QAM, s tim što je forma 256QAM dostupna samo u DVB-T2 standardu. 
Bitska brzina izvornog video signala je konstantna i u zavisnosti od korišćenog modulacionog formata izvorni video signal može imati bitsku brzinu i od nekoliko Gb/s. Međutim, zavisno od sadržaja video-signala, bitska brzina komprimovanog signala se menja tokom vremena, pa ukoliko je potrebno da prenos bude bez gubitaka kvaliteta, potrebno ga je takvog i preneti. U jednom multipleksu, u nekom određenom vremenskom trenutku različiti TV programi imaju različite i nezavisne promene bitske brzine [5].

Statističko multipleksiranje zasnovano je na algoritmima kontrole brzine koji su koristili koncept povratne sprege. Ovo je proizvedeno od strane kodera kroz promene kompresije u informacionom bitskom toku. Generisana statistika se koristi kao ulazni parametri u procesu kontrole kodera pre kompresije slike. Ova statistička kodifikacija zajedno sa informacionim stanjem bafera iz kanala koriste se za dinamičku obradu raspodele bitske brzine u svakom enkoderu. Bitska brzina programa je proporcionalna odnosu između složenosti bilo kog programa i sume kompleksnosti za sve programe [6]:

$$
R_{t}=R_{\text {channel }}\left(\frac{X_{i}}{\sum_{i} X_{i}}\right)
$$

gde je $R_{i}$ brzina protoka programa $i, R_{\text {channel }}$ brzina kanala, a $X$ je kompleksnost $i$-tog programa [7].

Ukupna bitska brzina u multipleksu ima manju disperziju zahvaljujući primeni statističkog multipleksa i kodera za kompresiju, što dovodi do boljeg iskorišćenja kapaciteta UHF/VHF kanala, a time i do povećanja broja TV programa koji se u istom smeštaju. Iako je bitska brzina pojedinih TV programa promenljiva u toku vremena, ukupna bitska brzina u multipleksu je skoro konstantna zahvaljujući činjenici da su promene bitske brzine TV programa nezavisne i da se postiže statistički balans u multipleksu zahvaljujući velikom broju TV programa koji se može smestiti. Ukupna bitska brzina zavisi od bitske brzine pojedinačnih TV programa, pre svega od njihovog formata, odnosno od prirode videosadržaja koji se prenosi [5].

Korišćenjem statističkog multipleksa, kompresiono kodovanje sa konstantnim protokom - CBR (Constant Bit Rate) se menja u kodovanje sa promenljivim protokom - VBR (Variable Bit Rate). Bitska brzina koju je potrebno dodeliti jednom TV programu svodi se na srednju vrednost bitske brzine dotičnog TV programa zahvaljujući upotrebi principa statističkog multipleksiranja i VBR-a. S druge strane, primenom CBR-a, svaki TV program mora da sadrži kapacitet znatno veći nego što je srednja vrednost. Ovaj efekat naziva se Dobitak statističkog multipleksiranja, a grafik promene dobitka efikasnosti u zavisnosti od broja TV kanala u multipleksu prikazana je na Slici 1 [3].

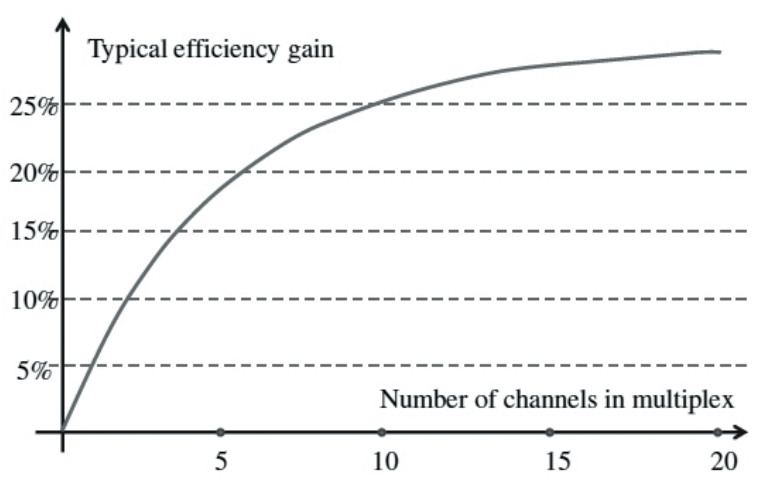

Slika 1. Dobitak u efikasnosti primenom statističkog multipleksiranja.

\section{STANDARDI ZA KOMPRESIJU TV SIGNALA}

Razvojem mnogobrojnih algoritama za video kompresiju i kompresionih standarda omogućeno je korišćenje standarda digitalne televizije standardne definicije SDTV (Standard Definition Television) i standarda digitalne televizije visoke definicije HDTV (High Definition Television). HDTV je standard koji daje bolji kvalitet slike i zvuka u odnosu na konzervativne pređašnje tehnologije za prikazivanje zvuka i slike (analogne PAL, NTSC, SECAM, i digitalne SDTV) [8], [9]. Poslednjih godina, pored HDTV javlja se televizija ultra visoke definicije koja uključuje 4K UHDTV (Ultra High Definition Television) i 8K UHDTV sa dva puta većim, odnosno četiri puta većim brojem piksela u odnosu na Full HDTV [10].

Dva najpopularnija kompresiona standarda za digitalne TV signale su MPEG-2 i MPEG-4 AVC (H.264) [8]. Za kompresiju HDTV-a uglavnom se koristi MPEG-4 standard dok su za kompresiju SDTV-a u upotrebi i MPEG-2 i MPEG-4. Za UHDTV od strane ITU (International Telecommunication Union) usvojen je kompresioni standard HEVC (High Efficiency Video Coding) [11].

U Tabeli 1 [12] dati su vrednosti protoka TV signala (bitska brzina) za SDTV, HDTV i UHDTV upotrebom različitih kompresionih standarda. 


\begin{tabular}{ccc}
\hline $\begin{array}{c}\text { Standardi za } \\
\text { kompresiju }\end{array}$ & $\begin{array}{c}\text { TV video } \\
\text { rezolucija }\end{array}$ & $\begin{array}{c}\text { TV bitska } \\
\text { brzina }[\mathbf{M b} / \mathbf{s}]\end{array}$ \\
\hline MPEG-2 & SDTV & 4 \\
\hline MPEG-2 & HDTV & 20 \\
\hline MPEG-4 (H.264) & SDTV & 2 \\
\hline MPEG-4 (H.264) & HDTV & 10 \\
\hline HEVC (H.265) & HDTV & 5 \\
\hline HEVC (H.265) & UHDTV & 30 \\
\hline
\end{tabular}

Tabela 1. Bitska brzina komprimovanog TV signala

U okviru TV signala prenose se i drugi podaci koji zauzimaju mali deo raspoložive bitske brzine u odnosu na video signal koji nesumnjivo zauzima najveći deo bitske brzine, a to su: audio, servisne informacije, EPG, teletekst, titlovi itd. Čiji ukupni protok ne prelazi više od $1 \mathrm{Mb} / \mathrm{s}$ [5].

\section{BITSKA BRZINA UHF KANALA}

U Tabeli 2 dati su primenjeni parametri za analizu bitske brzine jednog DVB-T2 multipleksa korišćenjem DVB kalkulatora [13].

\begin{tabular}{cc}
\hline Parametar & Vrednost \\
\hline Frame Duration & $250 \mathrm{~ms}$ \\
\hline Band Width & $8 \mathrm{MHz}$ \\
\hline Guide Interval & $1 / 128$ \\
\hline Pilot Pattern & PP7 \\
\hline FFT & $32 \mathrm{~K}$ \\
\hline
\end{tabular}

Tabela 2. Primenjeni parametri DVB-T2 za analizu

Koristeći DVB-T2 kalkulator izračunate su vrednosti bitske brzine jednog UHF kanala širine $8 \mathrm{MHz}$ za različite modulacione formate i različite vrednosti kodnog količnika. Rezultati su prikazani u Tabeli 3.

\begin{tabular}{ccccccc}
\hline \multirow{2}{*}{$\begin{array}{c}\text { Modu- } \\
\text { lacija }\end{array}$} & $\mathbf{1 / 2}$ & $\mathbf{3 / 5}$ & $\mathbf{2 / 3}$ & $\mathbf{3 / 4}$ & $\mathbf{4 / 5}$ & $\mathbf{5 / 6}$ \\
\cline { 2 - 7 } & $\mathbf{6}$ & $\mathbf{6}$ & & \\
QPSK & 7.38 & 8.87 & 9.91 & 11.11 & 11.85 & 12.37 \\
\hline 16QAM & 14.83 & 17.81 & 19.83 & 22.29 & 23.78 & 24.75 \\
\hline 64QAM & 22.21 & 26.68 & 29.74 & 33.39 & 35.63 & 37.19 \\
\hline 256QAM & 29.67 & 35.63 & 39.58 & 44.57 & 47.55 & 49.57 \\
\hline
\end{tabular}

Tabela 3. bitska brzina $[\mathrm{Mb} / \mathrm{s}]$ jednog UHF kanala
Na Slici 2 date su vrednosti AWGN-a (Additional White Gaussian Noise) jednog UHF kanala za različite vrednosti kodnog količnika i modulacionih formata $\mathrm{u}$ DVB-T2 standardu.

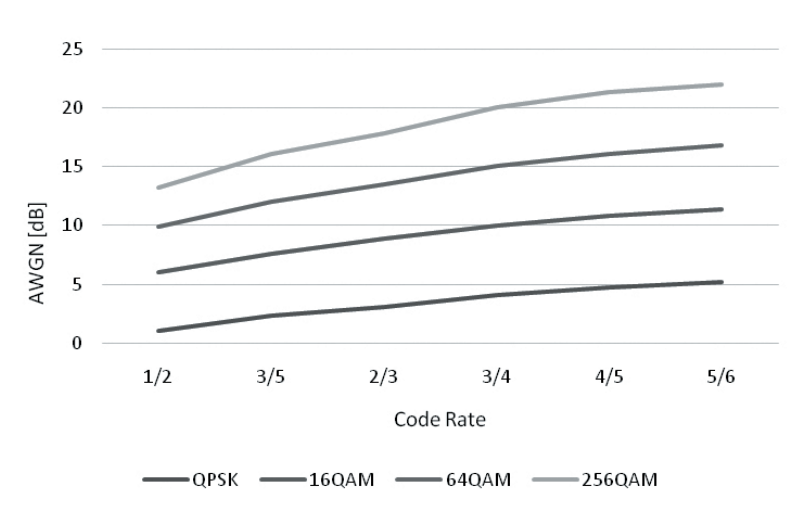

Slika 2. AWGN [dB] jednog UHF kanala.

QPSK format daje znatno niže vrednosti AWGN-a u poređenju sa drugim modulacionim formatima. Tako 256QAM modulacioni format omogućava četvorostruko veći AWGN od QPSK za najveću vrednost FEC parametra.

\section{POREĐENJE BROJA TV KANALA PRI KONSTANTNOJ I PROMENLJIVOJ BITSKOJ BRZINI}

Koristeći se podacima za bitsku brzinu jednog TV kanala iz Tabele $1 \mathrm{i}$ bitsku brzinu jednog UHF kanala za različite modulacione formate i kodne količnike datim u Tabeli 3, određen je broj TV kanala u multipleksu. Kako bi se uvideo uticaj statističkog multipleksa, određen je broj TV kanala u multipleksu korišćenjem promenljive i konstantne bitske brzine. Na Slici 3, Slici 4, Slici 5, Slici 6, Slici 7 i Slici 8 dat je maksimalni broj SDTV (u MPEG-2 formatu), SDTV (u MPEG-4 formatu), HDTV (u MPEG-2 formatu), HDTV (u MPEG-4 formatu), HDTV (u HEVC formatu) i UHDTV (u HEVC formatu) jednog UHF kanala širine $8 \mathrm{MHz}$, respektivno. Dat je broj TV kanala za CBR i VBR. Za referentne vrednosti bitske brzine TV kanala za različite kompresione standarde korišćene su vrednosti date u Tabeli 1, a dobitak u efikasnosti statističkog multipleksiranja je korišćen sa Slici 1. 
SDTV (MPEG-2)

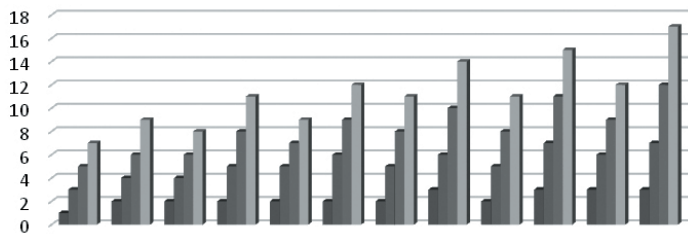

$\begin{array}{lllllllllllllllll}\text { CBR } & \text { VBR } & \text { CBR } & \text { VBR } & \text { CBR } & \text { VBR } & \text { CBR } & \text { VBR } & \text { CBR } & \text { VBR } & \text { CBR } & \text { VBR }\end{array}$

$1 / 2$

Slika 3. Broj SDTV kanala u MPEG-2 kompresionom standardu u jednom DVB-T2 multipleksu.

SDTV (MPEG-4)

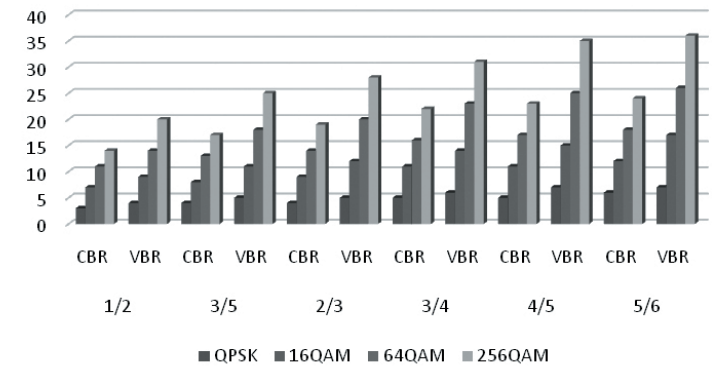

Slika 4. Broj SDTV kanala u MPEG-4 kompresionom standardu u jednom DVB-T2 multipleksu.

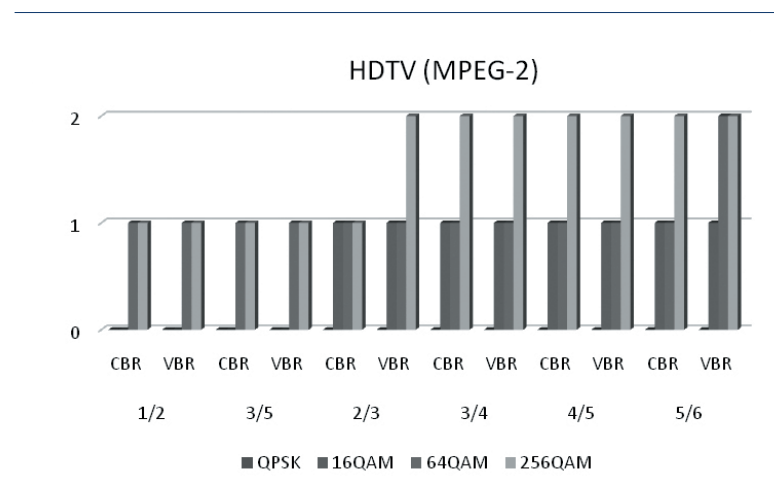

Slika 5. Broj HDTV kanala u MPEG-2 kompresionom standardu u jednom DVB-T2 multipleksu.

HDTV (MPEG-4)

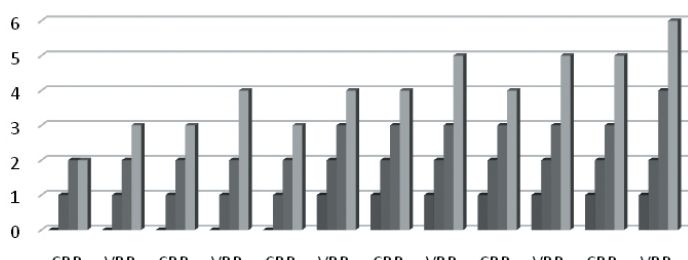

CBR VBR CBR VBR CBR VBR CBR VBR CBR VBR CBR VBR

$$
\begin{array}{llllll}
1 / 2 & 3 / 5 & 2 / 3 & 3 / 4 & 4 / 5 & 5 / 6
\end{array}
$$

- OPSK $=16 \mathrm{QAM}=64 \mathrm{QAM}=256 \mathrm{QAM}$

Slika 6. Broj HDTV kanala u MPEG-4 kompresionom standardu u jednom DVB-T2 multipleksu.
HDTV (HEVC)

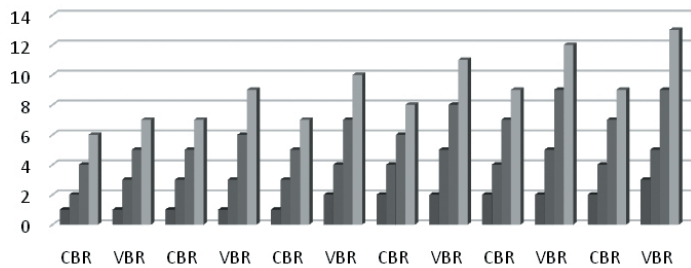

$1 / 2$

$3 / 5$

$2 / 3$

-QPSK $\backsim 16 \mathrm{QAM} \backsim 64 \mathrm{QAM} \backsim 256 \mathrm{QAM}$

Slika 7. Broj HDTV kanala u HEVC kompresionom standardu u jednom DVB-T2 multipleksu.

Sa datih slika se može videti da se najveći broj TV kanala može smestiti u jednom UHF kanalu koristeći 256QAM modulacionu šemu. Takođe, može se videti da je uticaj VBR znatno visok kod SDTV kanala i u MPEG2 i u MPEG-4 kompresionom formatu, kao i kod HDTV u HEVC kompresionom formatu. Broj UHDTV kanala je uglavnom isti korišćenjem i VBR i CBR, pre svega zato što je u pitanju zahtevana visoka bitska brzina i mali broj TV kanala koji se smeštaju u multipleksu nad kojima se primenjuje statistički multipleks.

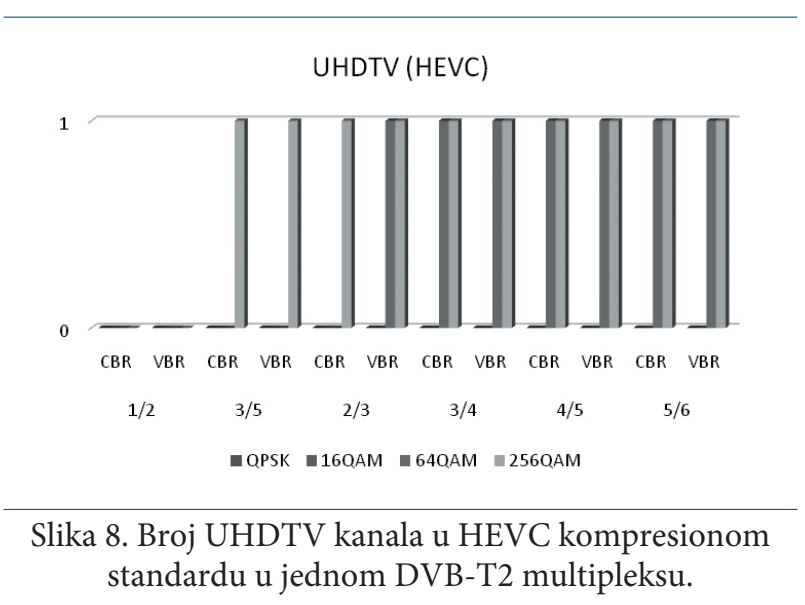

\section{OPTEREĆENJE DVB-T/T2 MULTIPLEKSA U ZEMLJAMA REGIONA}

U Tabeli 4 dat je pregled osnovnih parametara i realno stanje DVB-T/T2 multipleksa u Srbiji i zemljama u regionu [14-24]. Iz priloženih podataka može se videti da je kod DVB-T standarda zastupljen MPEG-4 kompresioni standard sa 64QAM modulacijom, a kod DVBT2 standarda 256QAM modulaciona tehnika. Promenljivi protok je zastupljen uglavnom kod svih multipleksa, osim kod onih sa MPEG-2 kompresionim standardom. Takođe, može se videti da je opterećenje multipleksa u okvirima granica dozvoljenih protoka za TV kanale 
prikazane u Tabeli 1. Deo multipleksa je sa slobodnim prostorom dok u pojedinim multipleksima (Crna Gora - MUX 2 i Mađarska - MUX E) opterećenje je veliko tako da je protok TV kanala u njima ispod granica prikazanih u Tabeli 1.



SRBIJA

\begin{tabular}{cccccccccccc}
\hline 1 & DVB-T2 & MPEG-4 & 256QAM & $2 / 3$ & $1 / 16$ & $32 \mathrm{k}$ & 36.82 & 15 & - & 5 & $\mathrm{~V}$ \\
\hline 3 & DVB-T2 & MPEG-4 & 256QAM & $3 / 4$ & $19 / 256$ & $32 \mathrm{k}$ & 41.5 & 26 & - & - & $\mathrm{V}$ \\
\hline \multicolumn{7}{c}{} & & \multicolumn{7}{c}{ HRVATSKA } \\
\hline A & DVB-T & MPEG-2 & 64QAM & $3 / 4$ & $1 / 4$ & $8 \mathrm{k}$ & 22.39 & 4 & - & - & $\mathrm{C}$ \\
\hline B & DVB-T & MPEG-2 & 64QAM & $3 / 4$ & $1 / 4$ & $8 \mathrm{k}$ & 22.39 & 4 & - & - & $\mathrm{C}$ \\
\hline D & DVB-T & MPEG-2 & 64QAM & $3 / 4$ & $1 / 4$ & $8 \mathrm{k}$ & 22.39 & 5 & - & - & $\mathrm{C}$ \\
\hline C & DVB-T2 & MPEG-4 & 256QAM & $2 / 3$ & $19 / 128$ & $32 \mathrm{k}$ & 36.52 & 27 & - & - & $\mathrm{V}$ \\
\hline E & DVB-T2 & MPEG-4 & 256QAM & $2 / 3$ & $19 / 128$ & $32 \mathrm{k}$ & 36.52 & 28 & - & 4 & $\mathrm{~V}$ \\
\hline
\end{tabular}

CRNA GORA

\begin{tabular}{cccccccccccc}
\hline 1 & DVB-T2 & MPEG-4 & 256QAM & $2 / 3$ & $1 / 16$ & $32 \mathrm{k}$ & 36.82 & 5 & - & 2 & $\mathrm{~V}$ \\
\hline 2 & DVB-T2 & MPEG-4 & 256QAM & $2 / 3$ & $1 / 16$ & $32 \mathrm{k}$ & 36.82 & 35 & - & - & $\mathrm{V}$ \\
\hline \multicolumn{10}{c}{ SLOVENIJA } \\
\hline
\end{tabular}

\begin{tabular}{ccccccccccccc}
\hline A & DVB-T & MPEG-4 & 64QAM & $2 / 3$ & $1 / 4$ & $8 \mathrm{k}$ & 19.91 & 4 & 2 & - & $\mathrm{V}$ \\
\hline $\mathrm{C}$ & DVB-T & MPEG-4 & 64QAM & $3 / 4$ & $1 / 4$ & $8 \mathrm{k}$ & 22.39 & 11 & - & - & $\mathrm{V}$ \\
\hline
\end{tabular}

\begin{tabular}{cccccccccccc}
\hline A & DVB-T & MPEG-4 & 64QAM & $3 / 4$ & $1 / 4$ & $8 \mathrm{k}$ & 22.39 & 1 & 3 & 4 & V \\
\hline B & DVB-T & MPEG-4 & 64QAM & $3 / 4$ & $1 / 4$ & $8 \mathrm{k}$ & 22.39 & 16 & - & - & V \\
\hline C & DVB-T & MPEG-4 & 64QAM & $3 / 4$ & $1 / 4$ & $8 \mathrm{k}$ & 22.39 & 6 & 2 & - & V \\
\hline D & DVB-T & MPEG-4 & 64QAM & $3 / 4$ & $1 / 4$ & $8 \mathrm{k}$ & 22.39 & 17 & - & - & $\mathrm{V}$ \\
\hline E & DVB-T & MPEG-4 & 64QAM & $3 / 4$ & $1 / 4$ & $8 \mathrm{k}$ & 22.39 & 20 & - & - & $\mathrm{V}$
\end{tabular}

\begin{tabular}{ccccccccccccc}
\hline \multicolumn{11}{c}{ RUMUNIJA } \\
\hline 1 & DVB-T2 & MPEG-4 & 256QAM & $3 / 4$ & $1 / 8$ & $32 \mathrm{k}$ & 22.39 & 8 & 1 & - & $\mathrm{V}$
\end{tabular}

\section{BUGARSKA}

\begin{tabular}{lllllllllllll}
\hline 1 & DVB-T & MPEG-4 & 64QAM & $3 / 4$ & $1 / 4$ & $8 \mathrm{k}$ & 22.39 & 5 & 1 & - & $\mathrm{V}$ \\
\hline 2 & DVB-T & MPEG-4 & 64QAM & $3 / 4$ & $1 / 4$ & $8 \mathrm{k}$ & 22.39 & 6 & - & 2 & $\mathrm{~V}$ \\
\hline
\end{tabular}

Tabela 4. Parametri i realno stanje DVB-T/T2 multipleksa u zemljama regiona

\section{ZAKLJUČAK}

Bitska brzina jednog UHF kanala je u direktnoj zavisnosti od njegovog frekvencijskog opsega i tipa modulacione šeme. Najveći broj TV kanala se smešta u DVB-T2 multipleksu korišćenjem 256QAM modulacione šeme. Primenom VBR omogućuje se smeštanje većeg broja TV kanala nego upotrebom CBR. Efekat VBR je znatno veći u slučajevima kada se koristi veći broj TV kanala u multipleksu, korišćenjem MPEG-4 kompresionog standarda za SDTV i HDTV kao i korišćenjem HEVC kompresionog standarda za HDTV. Zbog zahtevane velike bitske brzine, broj UHDTV kanala koji se smešta u multipleksu je mali pa je mala razlika između upotrebe CBR-a i VBR-a. 


\section{ZAHVALNOST}

Ovaj rad je rađen u okviru projekta Implementation of the study program - Digital Broadcasting and Broadband Technologies (DBBT Master studies), Erasmus+ CBHE Project No. 561688-EPP-1-2015-1-XK-EPPKA2CBHE-JP.

\section{LITERATURA}

[1] ETSI standard EN 302 755: Frame structure channel coding and modulation for a second generation digital terrestrial television broadcasting system (DVB-T2), EBU, 2009.

[2] M. Milivojevic, B. Miskovic, and I. Reljin, IEEE "Possible scenarios of HDTV and UHDTV programmes broadcasting within DVB-T2," in Proc. of 22nd Telecommunications forum TELFOR 2014, Belgrade, Serbia, November 25-27, 2014, pp. 897900

[3] K. McCann, and A. Mattei, Technical Evolution of the DTT Platform. London: ZetaCast, 2012.

[4] M. Rezaei, I. Bouazizi, and M. Gabbouj, "Statistical Time-Frequency Multiplexing of HD Video Traffic in DVB-T2," International Journal of Digital Multimedia Broadcasting, vol. 2009, Article ID 186960, pp 1-12, 2009.

[5] B. M. Mišković, "Povećanje broja programa u multipleksima DVB-T2 sistema," doktorska disertacija, Elektrotehnički fakultet, Univerzitet u Beogradu, Beograd, Srbija, 2015.

[6] K. Rao, Z. Bojkovic, and D. Milovanovic, "Joint video encoding in dvb/atsc multi-program transmission: Mpeg-2 vs. h.264/avc bitrate control," in Proc. 9th International Conference Telecommunication in Modern Satellite, Cable, and Broadcasting Services - TELSIKS, Nis, Serbia, 2009, pp. 3-12.
[7] M. Mirabito, and B. Morgenstern, Satellites: Operations and Applications. The New Communication Technologies. Burlington: Focal Press, 2004.

[8] S. Pechard, M. Carnec, P. Le Callet, and D. Barba, "From SD to HD television: effects of H.264 distortions versus display size on quality of experience," in Proc. of IEEE International Conference on Image Processing, Atlanta, USA, 2006, pp. 409-412.

[9] S. Haykin, Telecommunication Systems and Technology Vol.1: Analog and digital transmission of data..

[10] S. Marcotte, The road to UHDTV, Qubeck, Miranda Technologies, 2012.

[11] G. Cox G. "An Introduction to Ultra HDTV and HEVC," Report by ATEME, Paris, France, July 2013.

[12] R. Brugger R., and A. -I. Gbenga, "Spectrum usage and requirements of future terrestrial broadcast applications", Institut für Rundfunktechnik, München - EBU Technical Review 2009 Q4, EBU, Geneva, Okt. 2009.

[13] DVB Calculator: http://teamcast.com/

[14] Commission for Communications Regulation (CRC): http://www.crc.bg/

[15] Predajnici i veze: www.rdc.co.me

[16] TV za sve: http://www.tvzasve.me

[17] Odasiljaci i veze (OiV): http://www.oiv.hr/

[18] Evo TV: http://www.evotv.hr/

[19] Antenna Hungária: http://ahrt.hu/

[20] MinDig TV: http://mindigtv.hu/

[21] DVB-T Romania: http://www.radiocom.ro

[22] DVB-T Slovenia: http://dvb-t.akos-rs.si/

[23] Emisiona tehnika i veze: http://www.etv.rs/

[24] MTS Antena TV: https://mtsantenatv.rs/kanali. html 